\title{
Overall Evolution of Jetted Gamma-ray Burst Ejecta
}

\author{
Y. F. Huang, L. J. Gou, Z. G. Dai, and T. Lu ${ }^{1}$ \\ Department of Astronomy, Nanjing University, Nanjing 210093, P. R. China; \\ tlu@nju.edu.cn
}

Received —

Submitted to: The Astrophysical Journal

\footnotetext{
${ }^{1}$ Also at: China Center of Advanced Science and Technology (World Laboratory), P.O.Box.8730, Beijing 100080, P. R. China; Laboratory of Cosmic-Ray and High-Energy Astrophysics, Institute for High-Energy Physics, Chinese Academy of Sciences, Beijing 100039, P. R. China
} 


\begin{abstract}
Whether gamma-ray bursts are highly beamed or not is a very difficult but important problem that we are confronted with. Some theorists suggest that beaming effect usually leads to a sharp break in the afterglow light curve during the ultra-relativistic phase, with the breaking point determined by $\gamma=1 / \theta_{0}$, where $\gamma$ is the Lorentz factor of the blastwave and $\theta_{0}$ is the initial half opening angle of the ejecta, but numerical studies tend to reject the suggestion. We note that previous studies are uniformly based on dynamics that is not proper for non-relativistic blastwaves. Here we investigate the problem in more detail, paying special attention to the transition from the ultra-relativistic phase to the non-relativistic phase. Due to some crucial refinements in the dynamics, we can follow the overall evolution of a realistic jet till its velocity is as small as $\beta c \sim 10^{-3} c$. We find no obvious break in the optical light curve during the relativistic phase itself. However, an obvious break does appear at the transition from the relativistic phase to the Newtonian phase if the physical parameters involved are properly assumed. Generally speaking, the Newtonian phase is characterized by a sharp decay of optical afterglows, with the power law timing index $\alpha \sim 1.8-2.1$. This is due to the quick lateral expansion at this stage. The quick decay of optical afterglows from GRB 970228, 980326, and 980519, and the breaks in the optical light curves of GRB 990123 and 990510 may indicate the presence of highly collimated $\gamma$-ray burst ejecta.
\end{abstract}

Subject headings: gamma rays: bursts — ISM: jets and outflows hydrodynamics — radiation mechanisms: non-thermal — relativity 


\section{Introduction}

Till the end of August 1999, X-ray, optical, and radio afterglows have been observed from about 16, 11, and 5 gamma-ray bursts (GRBs) respectively (Costa et al. 1997; Bloom et al. 1998; Groot et al. 1998; Kulkarni et al. 1998, 1999; Harrison et al. 1999; Stanek et al. 1999; Fruchter et al. 1999; Galama et al. 1999a). The so called fireball model (Goodman 1986; Paczyński 1986; Mészáros \& Rees 1992; Rees \& Mészáros 1992, 1994; Katz 1994;

Sari, Narayan, \& Piran 1996; Dermer \& Mitman 1999; Dermer, Böttcher, \& Chiang 1999, 2000; Dermer 2000) is strongly favored, which is found successful at explaining the major features of GRB afterglows (Mészáros \& Rees 1997; Vietri 1997; Tavani 1997; Waxman 1997a; Wijers, Rees, \& Mészáros 1997; Sari 1997a; Huang et al. 1998a, b, 1999a, b; Dai \& Lu 1998a, b, c; Dermer, Chiang, \& Böttcher 1999; Dermer, Chiang, \& Mitman 2000). However, we are still far from resolving the puzzle of GRBs, because their "inner engines" are well hidden from direct afterglow observations.

To unveil the nature of the "inner engine", we first need to know the energetics involved in a typical burst, which itself depends on two factors: (i) the distance scale of GRBs, this has been settled since the BeppoSAX discovery of GRB 970228; (ii) the opening angle of GRB ejecta, i.e., whether gamma-rays are radiated isotropically or not, this question is still largely uncertain. Most GRBs localized by BeppoSAX have indicated isotropic energies of $10^{51}-10^{52} \mathrm{ergs}$, well within the energy output from compact stellar objects of solar-mass. However, GRB 971214, 980703, 990123, and 990510 have implied

isotropic gamma-ray releases of $3.0 \times 10^{53} \mathrm{ergs}\left(0.17 M_{\odot} c^{2}\right.$, Kulkarni et al. 1998), $1.0 \times 10^{53}$ $\operatorname{ergs}\left(0.06 M_{\odot} c^{2}\right.$, Bloom et al. 1998), $3.4 \times 10^{54} \operatorname{ergs}\left(1.9 M_{\odot} c^{2}\right.$, Kulkarni et al. 1999; Andersen et al. 1999), and $2.9 \times 10^{53} \operatorname{ergs}\left(0.16 M_{\odot} c^{2}\right.$, Harrison et al. 1999) respectively. Moreover, if really located at a redshift of $z \geq 5$ as suggested by Reichart et al. (1999), GRB 980329 would imply an isotropic gamma-ray energy of $5 \times 10^{54} \operatorname{ergs}\left(2.79 M_{\odot} c^{2}\right)$. 
Such enormous energetics has forced some theorists to deduce that GRB radiation must be highly collimated in these cases, with half opening angle $\theta \leq 0.2$, so that the intrinsic gamma-ray energy could be reduced by a factor of $10^{2}-10^{3}$, and could still come from compact stellar objects (Pugliese, Falcke, \& Biermann 1999). Obviously, whether GRBs are beamed or not is of fundamental importance to our understanding of their nature.

How can we tell a jet from an isotropic fireball? Gruzinov (1999) has argued that optical afterglows from a jet can be strongly polarized, in principle up to tens of percents, if co-moving magnetic fields parallel and perpendicular to the jet have different strengths and if we observe at the right time from the right viewing angle. More direct clues may come from afterglow light curves. Rhoads (1997, 1999a, b) has shown that the lateral expansion (at sound speed) of a relativistic jet (with a Lorentz factor $\gamma \geq 2$ ) will cause the blastwave to decelerate more quickly, leading to a sharp break in the afterglow light curve. The power law decay indices of afterglows from GRB 980326 and 980519 are anomalously large, $\alpha \sim 2.0$ (Groot et al. 1998; Owens et al. 1998), and optical light curves of GRB 990123 and 990510 even show obvious steepening at observing time $t \geq 1-2 \mathrm{~d}$ (Kulkarni et al. 1999; Harrison et al. 1999; Castro-Tirado et al. 1999). Recently GRB 970228 was also reported to have a large index of $\alpha \sim 1.73$ (Galama et al. 1999b). These phenomena have been widely regarded as the evidence of the presence of relativistic jets (Sari, Piran, \& Halpern 1999; Castro-Tirado et al. 1999).

However, numerical studies of some other authors (Panaitescu \& Mészáros 1998; Moderski, Sikora, \& Bulik 2000) have shown that due to the increased swept-up matter and the time delay of the large angle emission, the sideway expansion of the jet does not lead to an obvious dimming of the afterglow. Thus there are two opposite conclusions about the jet effect: the analytical solution predicts a sharp break, while the numerical calculation shows no such sharp breaks. It is very interesting to note that a recent analytic treatment 
by Wei \& Lu (2000) shows that the sharp break predicted by Rhoads is usually not exist unless the beaming angle is very small. This analysis seems to have given some supports to the numerical studies.

We find that previous studies on jet effects need to be improved urgently in the following three aspects: (i) Afterglows during the mildly relativistic $(2 \leq \gamma \leq 5)$ and non-relativistic $(\gamma \leq 1.5)$ phases of the expansion are of great importance to us, since they may correspond to observing time of $t \sim 2-5 \mathrm{~d}$ (Huang et al. 1998a, b). However, conventional dynamical model can not transit correctly from the ultra-relativistic phase $(\gamma \gg 1)$ to the non-relativistic phase for adiabatic shocks. This has been stressed by Huang, Dai, \& Lu (1999, 2000). Therefore previous numerical studies were based on improper dynamical equations. They could describe an ultra-relativistic jet, but they gave spurious results in the mildly relativistic and non-relativistic phases (Huang, Dai, \& Lu 1999a, b); (ii) It is reasonable to assume that the lateral expansion speed of the beamed ejecta is just the co-moving sound speed $c_{\mathrm{s}}$. Usually we take $c_{\mathrm{s}}=c / \sqrt{3}$, where $c$ is the speed of light (Rhoads 1997, 1999a). However in realistic case we expect $c_{\mathrm{s}}$ to vary with time, and especially it will by no means be $c / \sqrt{3}$ when $\gamma \sim 1$. This is another reason that previous studies are not proper for mildly relativistic and non-relativistic jets; (iii) In previous studies, the expansion of the beamed ejecta is supposed to be solely adiabatic. In fact the blastwave should be highly radiative at first. It evolves slowly into an adiabatic blastwave at later stages (Dai, Huang, \& Lu 1999).

This paper is aiming to overcome the problems mentioned just above. We present our refined dynamical model in Section 2. It can describe the overall evolution of a realistic, jetted GRB ejecta. Synchrotron radiation from shock accelerated electrons is formulated in Section 3. Our detailed numerical results are presented in Section 4. Section 5 is a brief discussion. 


\section{Dynamics}

\subsection{Basic Equations}

Let $R$ be the radial coordinate in the burster frame; $t_{\mathrm{b}}, t_{\mathrm{co}}$, and $t$ be the time from the event measured in the burster frame, co-moving ejecta frame, and terrestrial observer's frame respectively; $\gamma_{0}$ and $M_{\mathrm{ej}}$ be the initial Lorentz factor and ejecta mass and $\theta$ the half opening angle of the ejecta. The burst energy is $E_{0}=\gamma_{0} M_{\mathrm{ej}} c^{2}$.

It is useful to write down the following simple relations at first:

$$
\begin{gathered}
d R=\beta c d t_{\mathrm{b}} \\
d t_{\mathrm{b}}=\gamma d t_{\mathrm{co}}=\gamma\left(\gamma+\sqrt{\gamma^{2}-1}\right) d t
\end{gathered}
$$

where $\beta=\sqrt{\gamma^{2}-1} / \gamma$.

The evolution of radius $(R)$ and swept-up mass $(m)$ is described by

$$
\begin{gathered}
\frac{d R}{d t}=\beta c \gamma\left(\gamma+\sqrt{\gamma^{2}-1}\right), \\
\frac{d m}{d R}=2 \pi R^{2}(1-\cos \theta) n m_{\mathrm{p}},
\end{gathered}
$$

where $n$ is number density of surrounding interstellar medium (ISM) and $m_{\mathrm{p}}$ is mass of proton. The opening angle $\theta$ is usually evaluated as $\theta \approx a / R$, where $a$ is co-moving lateral radius of the ejecta (Rhoads 1999a; Moderski, Sikora, \& Bulik 2000). This is not ideal in numerical evaluations since $\theta$ may be as large as $0.5-0.8$ at later times. Here we use the following differential equation:

$$
\frac{d \theta}{d t} \equiv \frac{1}{R} \frac{d a}{d t}=\frac{c_{\mathrm{s}}\left(\gamma+\sqrt{\gamma^{2}-1}\right)}{R}
$$

As for the evolution of the Lorentz factor $\gamma$, the following equation has been widely used for both isotropic fireballs and beamed ejecta:

$$
\frac{d \gamma}{d m}=-\frac{\gamma^{2}-1}{M}
$$


where $M$ is the total mass in the co-moving frame, including internal energy $U$, $d M=[(1-\epsilon) \gamma+\epsilon] d m$ (Chiang \& Dermer 1999; Piran 1999; Moderski, Sikora, \& Bulik et al. 2000). Here $\epsilon$ is the radiative efficiency defined as the fraction of the shock-generated thermal energy (in the co-moving frame) that is radiated. $\epsilon=1$ corresponds to highly radiative case and $\epsilon=0$ corresponds to adiabatic expansion. However Huang, Dai, \& Lu (1999a, b) have demonstrated clearly that for adiabatic expansion equation (6) does not agree with the Sedov solution in the non-relativistic limit: equation (6) gives $\beta \propto R^{-3}$ while the Sedov solution requires $\beta \propto R^{-3 / 2}$ (Sedov 1969). They have proposed a new equation. Now we briefly repeat their derivation.

In the observer's frame, since the total kinetic energy of the fireball is $E_{\mathrm{k}}=(\gamma-1)\left(M_{\mathrm{ej}}+m\right) c^{2}+(1-\epsilon) \gamma U$ (Panaitescu, Mészáros, \& Rees 1998), and the radiated thermal energy is $\epsilon \gamma(\gamma-1) d m c^{2}$ (Blandford \& McKee 1976), we have

$$
d\left[(\gamma-1)\left(M_{\mathrm{ej}}+m\right) c^{2}+(1-\epsilon) \gamma U\right]=-\epsilon \gamma(\gamma-1) d m c^{2}
$$

For the item $U$, it is usually assumed that $d U=(\gamma-1) d m c^{2}$ (Panaitescu, Mészáros, \& Rees 1998). Equation (6) has been derived just in this way. Huang, Dai, \& Lu (1999a, b) suggested that $U=(\gamma-1) m c^{2}$ is a better approximation that makes equation (7) suitable for both ultra-relativistic and Newtonian shocks. Using this expression, it is easy to obtain (Huang, Dai, \& Lu 1999a, b)

$$
\frac{d \gamma}{d m}=-\frac{\gamma^{2}-1}{M_{\mathrm{ej}}+\epsilon m+2(1-\epsilon) \gamma m}
$$

Huang, Dai, \& Lu (1999a, b) have shown that this equation is acceptable for both radiative and adiabatic fireballs, and in both ultra-relativistic and non-relativistic phases. Here we use this equation to follow the overall evolution of beamed GRB ejecta.

Equations (3), (4), (5), and (8) present a thorough description of jet evolution. But before evaluating them numerically, we should give the expression for $c_{\mathrm{s}}$ and $\epsilon$. 


\subsection{Sound Speed}

The lateral expansion is determined by the co-moving sound speed. The simple assumption of $c_{\mathrm{s}}=c / \sqrt{3}$ is not proper for our purpose in this paper. We must derive $c_{\mathrm{s}}$ from $c_{\mathrm{s}}^{2} \equiv\left(d p^{\prime} / d e^{\prime}\right)_{\mathrm{s}}$, where $p^{\prime}$ and $e^{\prime}$ are co-moving pressure and energy density respectively. Kirk \& Duffy (1999) have derived

$$
c_{\mathrm{s}}^{2}=\frac{\hat{\gamma} p^{\prime}}{\rho^{\prime}}\left[\frac{(\hat{\gamma}-1) \rho^{\prime}}{(\hat{\gamma}-1) \rho^{\prime}+\hat{\gamma} p^{\prime}}\right] c^{2},
$$

where $\rho^{\prime}$ is co-moving mass density, and $\hat{\gamma}$ is the adiabatic index. Dai, Huang, \& Lu (1999) gave a simple and useful expression for $\hat{\gamma}, \hat{\gamma}=(4 \gamma+1) /(3 \gamma)$. Since $e^{\prime}=\gamma \rho^{\prime} c^{2}$ and $p^{\prime}=(\hat{\gamma}-1)\left(e^{\prime}-\rho^{\prime} c^{2}\right)$, it is easy to get

$$
c_{\mathrm{s}}^{2}=\hat{\gamma}(\hat{\gamma}-1)(\gamma-1) \frac{1}{1+\hat{\gamma}(\gamma-1)} c^{2} .
$$

In the ultra-relativistic limit $(\gamma \gg 1, \hat{\gamma} \approx 4 / 3)$, equation (10) gives $c_{\mathrm{s}}^{2}=c^{2} / 3$; and in the non-relativistic limit $(\gamma \sim 1, \hat{\gamma} \approx 5 / 3)$, we simply get $c_{\mathrm{s}}^{2}=5 \beta^{2} c^{2} / 9$. So equation (10) is a reasonable expression and will be used in our model.

\subsection{Radiative Efficiency}

As usual we assume that the magnetic energy density in the co-moving frame is a fraction $\xi_{\mathrm{B}}^{2}$ of the total thermal energy density (Dai, Huang, \& Lu 1999)

$$
\frac{B^{\prime 2}}{8 \pi}=\xi_{\mathrm{B}}^{2} \frac{\hat{\gamma} \gamma+1}{\hat{\gamma}-1}(\gamma-1) n m_{\mathrm{p}} c^{2},
$$

and that the shock accelerated electrons carry a fraction $\xi_{\mathrm{e}}$ of the proton energy. Here $\xi_{\mathrm{e}}$ is a parameter characterizing the efficiency of energy transport from protons to electrons. In realistic case, it is probable that $\xi_{\mathrm{e}}$ may vary with time, but little is known about the detailed mechanism. So we will take $\xi_{\mathrm{e}}$ as a constant throughout this article. This implies 
that the minimum Lorentz factor of the random motion of electrons in the co-moving frame is

$$
\gamma_{\mathrm{e}, \min }=\xi_{\mathrm{e}}(\gamma-1) \frac{m_{\mathrm{p}}(p-2)}{m_{\mathrm{e}}(p-1)}+1,
$$

where $m_{\mathrm{e}}$ is electron mass and $p$ is the index characterizing the power law energy distribution of electrons. We here consider only synchrotron emission from these electrons, and neglect the contribution of inverse Compton emission because the latter is unimportant particularly at late times (Waxman 1997a). The energy of a typical electron is lost due to both synchrotron radiation and expansion of the ejecta, thus the radiative efficiency of

this single electron is given by $t_{\mathrm{syn}}^{\prime-1} /\left(t_{\mathrm{syn}}^{\prime-1}+t_{\mathrm{ex}}^{\prime-1}\right)$ (Dai, Huang, \& Lu 1999), where $t_{\mathrm{syn}}^{\prime}$ is the synchrotron cooling time, $t_{\mathrm{syn}}^{\prime}=6 \pi m_{\mathrm{e}} c /\left(\sigma_{\mathrm{T}} B^{\prime 2} \gamma_{\mathrm{e}, \mathrm{min}}\right)$, with $\sigma_{\mathrm{T}}$ the Thompson cross section, and $t_{\mathrm{ex}}^{\prime}$ is the co-moving frame expansion time, $t_{\mathrm{ex}}^{\prime}=R /(\gamma c)$. Since all the shock accelerated electrons carry only a fraction $\xi_{\mathrm{e}}$ of the internal energy, the radiative efficiency of the total ejecta can be given by (Dai, Huang, \& Lu 1999)

$$
\epsilon=\xi_{\mathrm{e}} \frac{t_{\mathrm{syn}}^{\prime-1}}{t_{\mathrm{syn}}^{\prime-1}+t_{\mathrm{ex}}^{\prime-1}} .
$$

For the highly radiative expansion, $\xi_{\mathrm{e}} \approx 1$ and $t_{\mathrm{syn}}^{\prime} \ll t_{\mathrm{ex}}^{\prime}$, we have $\epsilon \approx 1$. The early evolution of the ejecta is likely in this regime. For an adiabatic expansion, $\xi_{\mathrm{e}} \ll 1$ or $t_{\mathrm{syn}}^{\prime} \gg t_{\mathrm{ex}}^{\prime}$, we get $\epsilon \approx 0$. The late evolution is believed to be in this regime. So one expects that in realistic case the radiative efficiency evolves from about 1 to 0 . In this paper we call the jet whose radiative efficiency evolves according to equation (13) a "realistic" one (Dai, Huang, \& Lu 1999).

\section{Synchrotron Radiation}




\subsection{Electron Distribution}

In the absence of radiation loss, the distribution of the shock accelerated electrons behind the blastwave is usually assumed to be a power law function of electron energy,

$$
\frac{d N_{\mathrm{e}}^{\prime}}{d \gamma_{\mathrm{e}}} \propto \gamma_{\mathrm{e}}^{-p}, \quad\left(\gamma_{\mathrm{e}, \min } \leq \gamma_{\mathrm{e}} \leq \gamma_{\mathrm{e}, \max }\right),
$$

where $\gamma_{\mathrm{e}, \max }$ is the maximum Lorentz factor, $\gamma_{\mathrm{e}, \max }=10^{8}\left(B^{\prime} / 1 \mathrm{G}\right)^{-1 / 2}$ (Dai, Huang, \& Lu 1999), and $p$ usually varies between 2 and 3. However, radiation loss may play an important role in the process. Electrons with different Lorentz factors have different radiation efficiencies. Sari, Piran, \& Narayan (1998) have derived an equation for the critical electron Lorentz factor, $\gamma_{c}$, above which synchrotron radiation is significant,

$$
\gamma_{\mathrm{c}}=\frac{6 \pi m_{\mathrm{e}} c}{\sigma_{\mathrm{T}} \gamma B^{\prime 2} t}
$$

Electrons with Lorentz factors below $\gamma_{\mathrm{c}}$ are adiabatic ones, and electrons above $\gamma_{\mathrm{c}}$ are highly radiative.

In the presence of steady injection of electrons accelerated by the shock, the distribution of radiative electrons becomes another power law function with an index of $p+1$ (Rybicki \& Lightman 1979), but the distribution of adiabatic electrons is unchanged. Then the actual distribution should be given according to the following cases (Dai, Huang, \& Lu 1999):

(i) For $\gamma_{\mathrm{c}} \leq \gamma_{\mathrm{e}, \min }$

$$
\begin{gathered}
\frac{d N_{\mathrm{e}}^{\prime}}{d \gamma_{\mathrm{e}}}=C_{1} \gamma_{\mathrm{e}}^{-(p+1)}, \quad\left(\gamma_{\mathrm{e}, \min } \leq \gamma_{\mathrm{e}} \leq \gamma_{\mathrm{e}, \max }\right), \\
C_{1}=\frac{p}{\gamma_{\mathrm{e}, \min }^{-p}-\gamma_{\mathrm{e}, \max }^{-p}} N_{\mathrm{ele}},
\end{gathered}
$$

where $N_{\text {ele }}$ is the total number of radiating electrons involved.

(ii) For $\gamma_{\mathrm{e}, \min }<\gamma_{\mathrm{c}} \leq \gamma_{\mathrm{e}, \max }$,

$$
\frac{d N_{\mathrm{e}}^{\prime}}{d \gamma_{\mathrm{e}}}= \begin{cases}C_{2} \gamma_{\mathrm{e}}^{-p}, & \left(\gamma_{\mathrm{e}, \min } \leq \gamma_{\mathrm{e}} \leq \gamma_{\mathrm{c}}\right) \\ C_{3} \gamma_{\mathrm{e}}^{-(p+1)}, & \left(\gamma_{\mathrm{c}}<\gamma_{\mathrm{e}} \leq \gamma_{\mathrm{e}, \max }\right)\end{cases}
$$


where

$$
\begin{gathered}
C_{2}=C_{3} / \gamma_{\mathrm{c}} \\
C_{3}=\left[\frac{\gamma_{\mathrm{e}, \min }^{1-p}-\gamma_{\mathrm{c}}^{1-p}}{\gamma_{\mathrm{c}}(p-1)}+\frac{\gamma_{\mathrm{c}}^{-p}-\gamma_{\mathrm{e}, \text { max }}^{-p}}{p}\right]^{-1} N_{\text {ele }}
\end{gathered}
$$

(iii) If $\gamma_{\mathrm{c}}>\gamma_{\mathrm{e}, \max }$, then

$$
\frac{d N_{\mathrm{e}}^{\prime}}{d \gamma_{\mathrm{e}}}=C_{4} \gamma_{\mathrm{e}}^{-p}, \quad\left(\gamma_{\mathrm{e}, \min } \leq \gamma_{\mathrm{e}} \leq \gamma_{\mathrm{e}, \max }\right)
$$

where

$$
C_{4}=\frac{p-1}{\gamma_{\mathrm{e}, \min }^{1-p}-\gamma_{\mathrm{e}, \max }^{1-p}} N_{\mathrm{ele}}
$$

\subsection{Relativistic Transformations}

In the co-moving frame, synchrotron radiation power at frequency $\nu^{\prime}$ from electrons is given by (Rybicki \& Lightman 1979)

$$
P^{\prime}\left(\nu^{\prime}\right)=\frac{\sqrt{3} e^{3} B^{\prime}}{m_{\mathrm{e}} c^{2}} \int_{\gamma_{\mathrm{e}, \min }}^{\gamma_{\mathrm{e}, \max }}\left(\frac{d N_{\mathrm{e}}^{\prime}}{d \gamma_{\mathrm{e}}}\right) F\left(\frac{\nu^{\prime}}{\nu_{\mathrm{c}}^{\prime}}\right) d \gamma_{\mathrm{e}}
$$

where $e$ is electron charge, $\nu_{\mathrm{c}}^{\prime}=3 \gamma_{\mathrm{e}}^{2} e B^{\prime} /\left(4 \pi m_{\mathrm{e}} c\right)$, and

$$
F(x)=x \int_{x}^{+\infty} K_{5 / 3}(k) d k
$$

with $K_{5 / 3}(k)$ being the Bessel function. We assume that this power is radiated isotropically,

$$
\frac{d P^{\prime}\left(\nu^{\prime}\right)}{d \Omega^{\prime}}=\frac{P^{\prime}\left(\nu^{\prime}\right)}{4 \pi}
$$

Let $\Theta$ be the angle between the velocity of emitting material and the line of sight and define $\mu=\cos \Theta$, we can derive the angular distribution of power in the observer's frame (Rybicki \& Lightman 1979),

$$
\frac{d P(\nu)}{d \Omega}=\frac{1}{\gamma^{3}(1-\beta \mu)^{3}} \frac{d P^{\prime}\left(\nu^{\prime}\right)}{d \Omega^{\prime}}=\frac{1}{\gamma^{3}(1-\beta \mu)^{3}} \frac{P^{\prime}\left(\nu^{\prime}\right)}{4 \pi},
$$




$$
\nu=\frac{\nu^{\prime}}{\gamma(1-\mu \beta)}
$$

Then the observed flux density at frequency $\nu$ is

$$
S_{\nu}=\frac{1}{A}\left(\frac{d P(\nu)}{d \Omega} \frac{A}{D_{\mathrm{L}}^{2}}\right)=\frac{1}{\gamma^{3}(1-\beta \mu)^{3}} \frac{1}{4 \pi D_{\mathrm{L}}^{2}} P^{\prime}(\gamma(1-\mu \beta) \nu) \text {, }
$$

where $A$ is the area of our detector and $D_{\mathrm{L}}$ is the luminosity distance.

\subsection{Equal Arrival Time Surfaces}

Photons received by the detector at a particular time $t$ are not emitted simultaneously in the burster frame. This effect has been emphasized by a number of authors (Waxman 1997b; Sari 1997b; Panaitescu \& Mészáros 1998) and may be of great importance to jet radiation (Moderski, Sikora, \& Bulik 2000; Panaitescu \& Mészáros 1999). In order to calculate observed flux densities, we should integrate over the equal arrival time surface determined by

$$
t=\int \frac{1-\beta \mu}{\beta c} d R \equiv \text { const }
$$

within the jet boundaries.

\section{Numerical Results}

For convenience, let us define the following initial values or parameters as a set of "standard" parameters: initial energy per solid angle $E_{0} / \Omega_{0}=10^{54} \mathrm{ergs} / 4 \pi, \gamma_{0}=300$ (i.e., initial ejecta mass per solid angle $\left.M_{\mathrm{ej}} / \Omega_{0}=0.001867 M_{\odot} / 4 \pi\right), n=1 \mathrm{~cm}^{-3}, \xi_{\mathrm{B}}^{2}=0.01$, $p=2.5, D_{\mathrm{L}}=1.0 \times 10^{6} \mathrm{kpc}, \xi_{\mathrm{e}}=1, \theta_{0}=0.2$. For simplicity, we first assume that the expansion is completely adiabatic all the time (i.e. $\epsilon \equiv 0$, we call it an "ideal" jet, distinguishing it from the "realistic" jet defined in Section 2.3). 
Figure 1 shows the evolution of the Lorentz factor. We see that the ultra-relativistic phase lasts only for $\sim 10^{5} \mathrm{~s}$, this is the period during which equation (6) can be safely applied. The mildly relativistic phase lasts from $\sim 10^{5} \mathrm{~s}$ to $\sim 10^{6.5} \mathrm{~s}$. In short, the ejecta will cease to be highly relativistic at time $t \sim 10^{5}-10^{6} \mathrm{~s}$. This clearly demonstrates the necessity to replace equation (6) with our improved expression of equation (8). Figure 1 also indicates that the transition from the relativistic phase to the non-relativistic phase occurs roughly at $t \sim 10^{6.5}-10^{7} \mathrm{~s}$. Figure 2 illustrates the time dependence of shock radius.

\section{EDITOR: PLACE FIGURE 1 HERE.}

\section{EDITOR: PLACE FIGURE 2 HERE.}

In Figure 3 we present the evolution of the jet opening angle $\theta$. During the ultra-relativistic phase $\left(t \leq 10^{5} \mathrm{~s}\right), \theta$ increases only slightly. But at the Newtonian stage $\left(t \geq 10^{7} \mathrm{~s}\right)$, the increase of $\theta$ is very quick. Again this figure indicates that the critical point is roughly at $t \sim 10^{6.5} \mathrm{~s}$. The usual approximation of $\theta \approx a / R$ is not a good approach and our equation (5) is obviously more reasonable. Figure 4 illustrates jet evolution on the $y-z$ plane schematically, where $z$-axis is just the symmetry axis of the jet and the lateral expansion is approximately at $y$ direction. Note that the jet is non-relativistic when $z \approx 3.2 \times 10^{18} \mathrm{~cm}$. Again we see the quick lateral expansion at the Newtonian stage. In Figure 5 we show some examples of equal arrival time surfaces on the $y-z$ plane. Here we assume that the angle between jet symmetry axis and the line of sight is $\theta_{\text {obs }}=0$.

EDITOR: PLACE FIGURE 3 HERE.

EDITOR: PLACE FIGURE @ HERE. 
EDITOR: PLACE FIGURE 5 HERE.

Figures 6 and 7 illustrate the time dependence of radiative efficiency $(\epsilon)$ and total kinetic energy $\left(E_{\mathrm{k}}\right)$ for "realistic" jets respectively. Although the highly radiative phase usually lasts for very short period $\left(t \leq 10^{4}-10^{5} \mathrm{~s}\right)$, it dissipates the total kinetic energy substantially (up to $90 \%$ in less than $10^{3} \mathrm{~s}$ ). This effect may account for the confusing

phenomena observed in some GRBs: $\gamma$-ray energy released in the initial GRB phase $\left(E_{\gamma}\right)$ is comparable to or even higher than the total kinetic energy inferred from afterglow observations (Wijers \& Galama 1999).

EDITOR: PLACE FIGURE 6 HERE.

EDITOR: PLACE FIGURE 7 HERE.

Figure 8 shows the optical light curves, computed for $\mathrm{R}$ band observation. In this figure, the thick solid line corresponds to an "ideal" jet with "standard" parameters, and viewing angle $\theta_{\mathrm{obs}}=0$. For comparison, other lines are drawn with only one parameter altered or only one condition changed. Please note that at end point of each curve, the average electron Lorentz factor is already as small as $\gamma_{\mathrm{e}} \sim 5$, corresponding to bulk Lorentz factor of $\gamma \approx 1.002$, thus completely in the Newtonian regime.

\section{EDITOR: PLACE FIGURE 8 HERE.}

¿From Figure 8, we see that in no case could we observe the theoretically predicted light curve steepening during the relativistic stage itself (i.e., when $t \leq 10^{6} \mathrm{~s}$ ), consistent with previous numerical studies (Panaitescu \& Mészáros 1999; Moderski, Sikora, \& Bulik 2000). 
This has been attributed to the effects of equal arrival time surfaces and more and more swept-up material. Here we would like to propose another more reasonable explanation. Rhoads is correct in saying that the lateral expansion begins to take effect when $\gamma \sim 1 / \theta_{0}$. In our calculations this occurs at $t \sim 10^{5.5} \mathrm{~s}$. However, the blastwave is already in its mildly relativistic phase at that moment and it will become non-relativistic soon after that (i.e., when $t \geq 10^{6.5} \mathrm{~s}$, see Figure 1). So it is not surprising that we could not see any obvious breaks during the relativistic phase, they just do not have time to emerge.

Fortunately we have pointed out that the lateral expansion is even more notable in the non-relativistic phase, so we expect that a break should occur in the light curve between the relativistic stage and the non-relativistic stage. The dash-dotted line in Figure 8 proves our deduction. This line corresponds to an "ideal" jet with $\xi_{\mathrm{e}}=0.1$ and with other parameters equal to those in the "standard" set. Here the decay in the relativistic phase is $S_{\mathrm{R}} \propto t^{-1.61}$, in good agreement with theoretical results for isotropic fireballs (Wijers, Rees, \& Mészáros 1997; Sari et al. 1998), and the decay in the non-relativistic phase is $S_{\mathrm{R}} \propto t^{-2.14}$, also consistent with Rhoads' prediction for lateral expansion effect (but made only for relativistic jets). Previous numerical studies are based on dynamical equations improper for non-relativistic blastwaves. They could not correctly reveal the break between relativistic and Newtonian stages (Panaitescu \& Mészáras 1999; Moderski, Sikora, \& Bulik 2000).

One may ask why other lines in Figure 8 do not show any breaks. The reason is they all correspond to $\xi_{\mathrm{e}}=1$ and their peaks appear at relatively late stages. For example, the thick solid line peaks at about $10^{5}$ s. Then we can not see the initial power law decay (i.e., $\alpha \sim 1.1)$ in the relativistic phase and can only see the fast decay of $S_{\mathrm{R}} \propto t^{-2.14}$ at lage stages. However, we should note that although they do not show any obvious breaks, they all have a common characteristic: a large timing index of $\alpha \sim 1.8-2.1$. We suggest that 
the sharp decline of afterglows from some GRBs (with $\alpha \sim 1.7-2.0$ ) itself may just be the evidence of the presence of a jet (Huang, Dai, \& Lu 2000a, b, c).

Dai \& Lu (1999, 2000) have discussed the optical afterglows from isotropic fireballs during the non-relativistic phase extensively. The most obvious difference between their results and ours is that our timing index at non-relativistic stage is reasonably larger.

\section{Conclusion and Discussion}

In this paper we investigate the detailed dynamical evolution of jets and their afterglows. Our model is simple in form and is easy for numerical evaluations. Comparing with previous studies, the model is refined in the following aspects:

(i) Equation (6) has been widely used in previous studies, however, it is not correct for non-relativistic ejecta (Huang, Dai, \& Lu 1999a, b). The dynamics here (i.e., mainly our equation (8) ) is applicable to both ultra-relativistic and Newtonian jets, so we could follow the overall evolution (from $\gamma \gg 1$ to $\gamma \sim 1$ ) of a jet by using a single set of differential equations. Numerical results indicate that the ejecta will cease to be ultra-relativistic $10^{5}-10^{6} \mathrm{~s}$ after the main GRB. We should consider this refinement seriously.

(ii) We describe the lateral expansion of jets with a refined sound speed expression, which gives reasonable approximations during both ultra-relativistic and Newtonian phases (Kirk \& Duffy 1999).

(iii) The remnant here is more "realistic", i.e., the radiative efficiency evolves from 1 (corresponding to highly radiative regime) to 0 (adiabatic regime), according to our equation (13). 
(iv) We use a differential equation to describe the increase of jet opening angle $\theta$, not simply using $\theta \equiv a / R$.

Our model also takes many other important factors into account, for example: the equal arrival time surface, the distribution of electrons, and the viewing angle.

We find that during the ultra-relativistic phase, the opening angle $\theta$ increases only slightly, but at the Newtonian stage it increases quickly to $\theta \sim 0.8-1$. The highly radiative regime (when $\epsilon \sim 1$ ) lasts usually for very short period, however a substantial fraction of the initial kinetic energy could be dissipated in less than $10^{2}-10^{3} \mathrm{~s}$. As for the light curves of optical afterglows, we find no theoretically predicted breaks at the critical points where the ejecta transits from $\gamma \geq 1 / \theta$ to $\gamma \leq 1 / \theta$, consistent with previous numerical studies (Panaitescu \& Mészáros 1999; Moderski, Sikora, \& Bulik 2000). The reason is that at this critical point the lateral expansion just begins to take effect, but its action does not prevail over $\theta_{0}$ completely, and also the relativistic phase is too short for the break to appear. However we have shown that the lateral expansion is even more striking during the non-relativistic phase, so we expect an obvious break between the relativistic stage and the non-relativistic stage. This has been proved by our calculations made with $\xi_{\mathrm{e}}=0.1$. Other calcuations made with $\xi_{\mathrm{e}}=1$ do not show any obvious breaks, but they are all characterized by a large timing index.

We conclude that the most obvious characteristic of beamed eject is the quick decline of afterglows at late stages (in fact corresponding to the non-relativistic phase), with $\alpha \sim 1.8$ - 2.1, and in some cases, we could even see a sharp break in the light curve between the relativistic stage and the non-relativistic stage. We suggest that the quick decay (with $\alpha \sim$ 1.7 - 2.0) of optical afterglows from some GRBs, such as GRB $970228(t \leq 10 \mathrm{~d}), 980326$, and 980519, and the breaks in the optical light curves of GRB 990123 and 990510, are probably due to beaming effect (Huang, Dai, \& Lu 2000a, b, c). 
But we should also keep in mind that other factors might lead to a large $\alpha$ as well. For example, the ISM density may be a decline function of radius (usually $n \propto R^{-2}$ ), then an isotropic fireball can well explain the quick decay of optical afterglows from GRB 970228, 980326, and 990519. Additionally, if an isotropic GRB remnant sweeps through a uniform ISM $\left(n \propto R^{0}\right)$ and an uneven ISM (i.e., $\left.n \propto R^{-2}\right)$ in succession, then an obvious break is likely to present in the optical light curve. GRB 990123 and 990510 may also correspond to this case. The suggestion by Dai \& Lu $(1999,2000)$ that the quick decay of afterglows from GRB 980519 and 990123 is due to an isotropic Newtonian blastwave in a dense ISM (with $n \sim 10^{6} \mathrm{~cm}^{-3}$ ) is also possible. So, the degree of beaming is still very difficult to determine now. With the progress in observing technique, when much more GRBs are localized, maybe we could also infer some hints from statistical researches. However the final solution may come from systematic deep optical surveys, which are expected to find many faint decaying optical sources if GRBs are highly collimated. They are afterglows from jetted GRBs whose gamma-ray emission deviates the line of sight slightly.

This work was partly supported by the National Natural Science Foundation of China, grants 19773007 and 19825109, the National Climbing Project on Fundamental Researches, and the National Project of Fundamental Researches (973 Project). 


\section{REFERENCES}

Andersen, M., et al. 1999, Science, 283, 2075

Blandford, R.D., \& McKee, C.F. 1976, Phys. Fluids, 19, 1130

Bloom, J.S., et al. 1998, ApJ, 508, L21

Castro-Tirado, A., et al. 1999, Science, 283, 2069

Chiang, J., \& Dermer, C.D. 1999, ApJ, 512, 699

Costa, E., et al. 1997, Nature, 387, 783

Dai, Z.G., Huang, Y.F., \& Lu, T. 1999, ApJ, 520, 634

Dai, Z.G., \& Lu, T. 1998a, MNRAS, 298, 87

Dai, Z.G., \& Lu, T. 1998b, A\&A, 333, L87

Dai, Z.G., \& Lu, T. 1998c, Phys. Rev. Lett., 81, 4301

Dai, Z.G., \& Lu, T. 1999, ApJ, 519, L155

Dai, Z.G., \& Lu, T. 2000, ApJ, in press, astro-ph/9906109

Dermer, C.D. 2000, ApJ submitted, astro-ph/0002306

Dermer, C.D., Böttcher, M., \& Chiang, J. 1999, ApJ, 515, L49

Dermer, C.D., Böttcher, M., \& Chiang, J. 2000, ApJ, 537, in press, astro-ph/9910472

Dermer, C.D., \& Mitman, K.E. 1999, ApJ, 513, L5

Dermer, C.D., Chiang, J., \& Böttcher, M. 1999, ApJ, 513, 656

Dermer, C.D., Chiang, J., \& Mitman, K.E. 2000, ApJ submitted, astro-ph/9910240 
Fruchter, A.S., et al. 1999, ApJ, 519, L13

Galama, T.J., et al. 1999a, Nature, 398, 394

Galama, T.J., et al. 1999b, ApJ submitted, astro-ph/9907264

Goodman, J. 1986, ApJ, 308, L47

Groot, P.J., et al. 1998, ApJ, 502, L123

Gruzinov, A. 1999, ApJ, 525, L29

Harrison, F.A., et al. 1999, ApJ, 523, L121

Huang, Y.F., Dai, Z.G., \& Lu, T. 1998a, A\&A, 336, L69

Huang, Y.F., Dai, Z.G., Wei, D.M., \& Lu, T. 1998b, MNRAS, 298, 459

Huang, Y.F., Dai, Z.G., \& Lu, T. 1999a, Chin. Phys. Lett., 16, 775 astro-ph/9906404)

Huang, Y.F., Dai, Z.G., \& Lu, T. 1999b, MNRAS, 309, 513 (astro-ph/9906370)

Huang, Y.F., Dai, Z.G., \& Lu, T. 2000a, A\&A, 355, L43 (astro-ph/0002433)

Huang, Y.F., Dai, Z.G., \& Lu, T. 2000b, MNRAS, in press

Huang, Y.F., Dai, Z.G., \& Lu, T. 2000c, Chin. Phys. Lett., in press

Katz, J. 1994, ApJ, 422, 248

Kirk, J.G., \& Duffy, P. 1999, Journal of Physics G, 25, R163

Kulkarni, S.R., et al. 1998, Nature, 393, 35

Kulkarni, S.R., et al. 1999, Nature, 398, 389

Mészáros, P., \& Rees, M.J. 1992, MNRAS, 257, 29P 
Mészáros, P., \& Rees, M.J. 1997, ApJ, 476, 232

Moderski, R., Sikora, M., \& Bulik, T. 2000, ApJ, 529, 151

Owens, A., et al. 1998, A\&A, 339, L37

Paczyński, B. 1986, ApJ, 308, L43

Panaitescu, A., \& Mészáros, P. 1998, ApJ, 493, L31

Panaitescu, A., \& Mészáros, P. 1999, ApJ, 526, 707

Panaitescu, A., Mészáros, P., \& Rees, M.J. 1998, ApJ, 503, 315

Piran, T. 1999, Phys. Rep., 314, 575

Pugliese, G., Falcke, H., \& Biermann, P.L. 1999, A\&A, 344, L37

Rees, M.J., \& Mészáros, P. 1992, MNRAS, 258, 41P

Rees, M.J., \& Mészáros, P. 1994, ApJ, 430, L93

Reichart, D.E., Lamb, D.Q., Metzger, M.R., Quashnock, J.M., Cole, D.M., Castander, F.J., Cooray, A.R., \& Vanden Berk, D.E. 1999, ApJ, 517, 692

Rhoads, J. 1997, ApJ, 487, L1

Rhoads, J. 1999a, ApJ, 525, 737

Rhoads, J. 1999b, A\&AS, 138, 539

Rybicki, G.B., \& Lightman, A.P. 1979, Radiative Processes in Astrophysics, Wiley, New York

Sari, R. 1997a, ApJ, 489, L37 
Sari, R. 1997b, ApJ, 494, L49

Sari, R., Narayan, R., \& Piran, T. 1996, ApJ, 473, 204

Sari, R., Piran, T., \& Halpern, J.P. 1999, ApJ, 519, L17

Sari, R., Piran, T., \& Narayan, R. 1998, ApJ, 497, L17

Sedov, L. 1969, Similarity and Dimensional Methods in Mechanics, Academic, New York, Ch. IV

Stanek, K.Z., Garnavich, P.M., Kaluzny, J., Pych, W., \& Thompson, I. 1999, ApJ, 522, L39

Tavani, M. 1997, ApJ, 483, L87

Vietri, M. 1997, ApJ, 488, L105

Waxman, E. 1997a, ApJ, 485, L5

Waxman, E. 1997b, ApJ, 491, L19

Wei, D.M., \& Lu, T. 2000, ApJ, in press

Wijers, R.A.M.J., \& Galama, T.J. 1999, ApJ, 523, 177

Wijers, R.A.M.J., Rees, M.J., \& Mészáros, P. 1997, MNRAS, 288, L51

This manuscript was prepared with the AAS LATEX macros v4.0. 
Fig. 1.- Evolution of the Lorentz factor $(\gamma)$. The solid line corresponds to an "ideal" jet with "standard" parameters. The dashed line is for "ideal" jet with $\theta_{0}=0.1$ and with other parameters being equal to those in the "standard" set. For the meaning of "ideal" and "standard", please see Section 4 in the main text.

Fig. 2.- Evolution of the shock radius $(R)$. Parameters and line styles are the same as in Fig. 1.

Fig. 3.- Evolution of the half opening angle $(\theta)$. Parameters and line styles are the same as in Fig. 1.

Fig. 4. - Schematic illustration of jet evolution on the $y-z$ plane. $Z$-axis is the symmetry axis of the jet and the lateral expansion is approximately at $y$ direction. Parameters and line styles are the same as in Fig. 1.

Fig. 5.- Schematic illustration of the equal arrival time surfaces on the $y-z$ plane. The jet is "ideal" and with "standard" parameters. The viewing angle is assumed to be $\theta_{\text {obs }}=0$. The dash-dotted lines from right to left correspond to spherical ejecta shells at observing time of $t=10^{8} \mathrm{~s}, 10^{7} \mathrm{~s}, 10^{6} \mathrm{~s}$, and $10^{5} \mathrm{~s}$ respectively, enclosed within the jet boundary (dotted lines). The solid lines are corresponding equal arrival time surfaces.

Fig. 6.- Evolution of the radiative efficiency $(\epsilon)$ for a "realistic" jet with "standard" parameters.

Fig. 7.- Time dependence of the total kinetic energy $\left(E_{\mathrm{k}}\right)$ of a "realistic" jet. Parameters are the same as in Fig. 6.

Fig. 8.- R band afterglows. $S_{\mathrm{R}}$ is in units of $\operatorname{ergs} \cdot \mathrm{s}^{-1} \cdot \mathrm{Hz}^{-1} \cdot \mathrm{cm}^{-2}$. The thick solid line corresponds to an "ideal" jet with "standard" parameters, and viewing angle $\theta_{\text {obs }}=0$. Other lines are drawn with one parameter altered or one condition changed: the thin solid 
line corresponds to $\theta_{\mathrm{obs}}=0.3$; the dashed line corresponds to $\theta_{0}=0.1$; the dash-dotted line corresponds to $\xi_{\mathrm{e}}=0.1$; and the dotted line is for a "realistic" jet. 


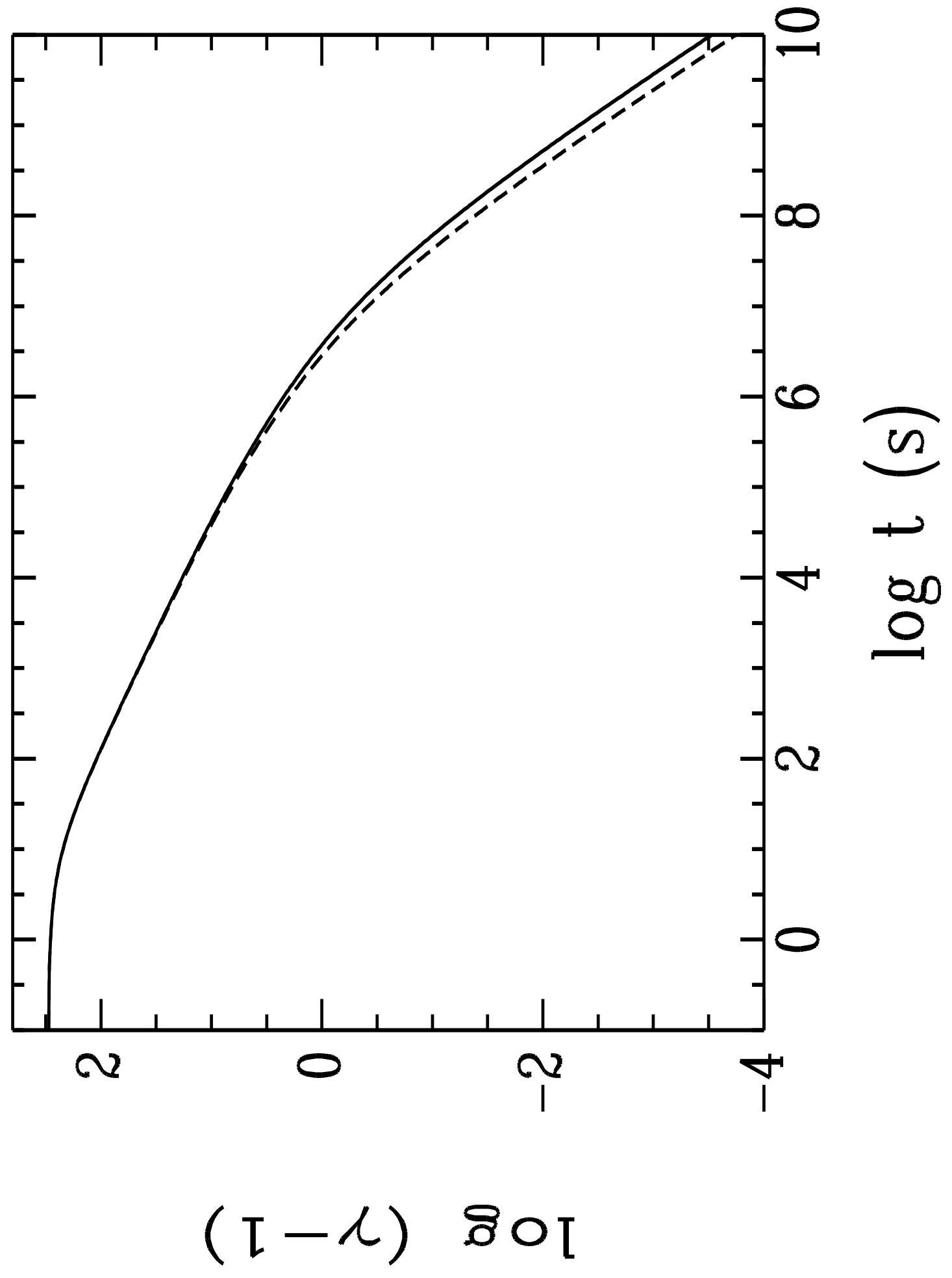




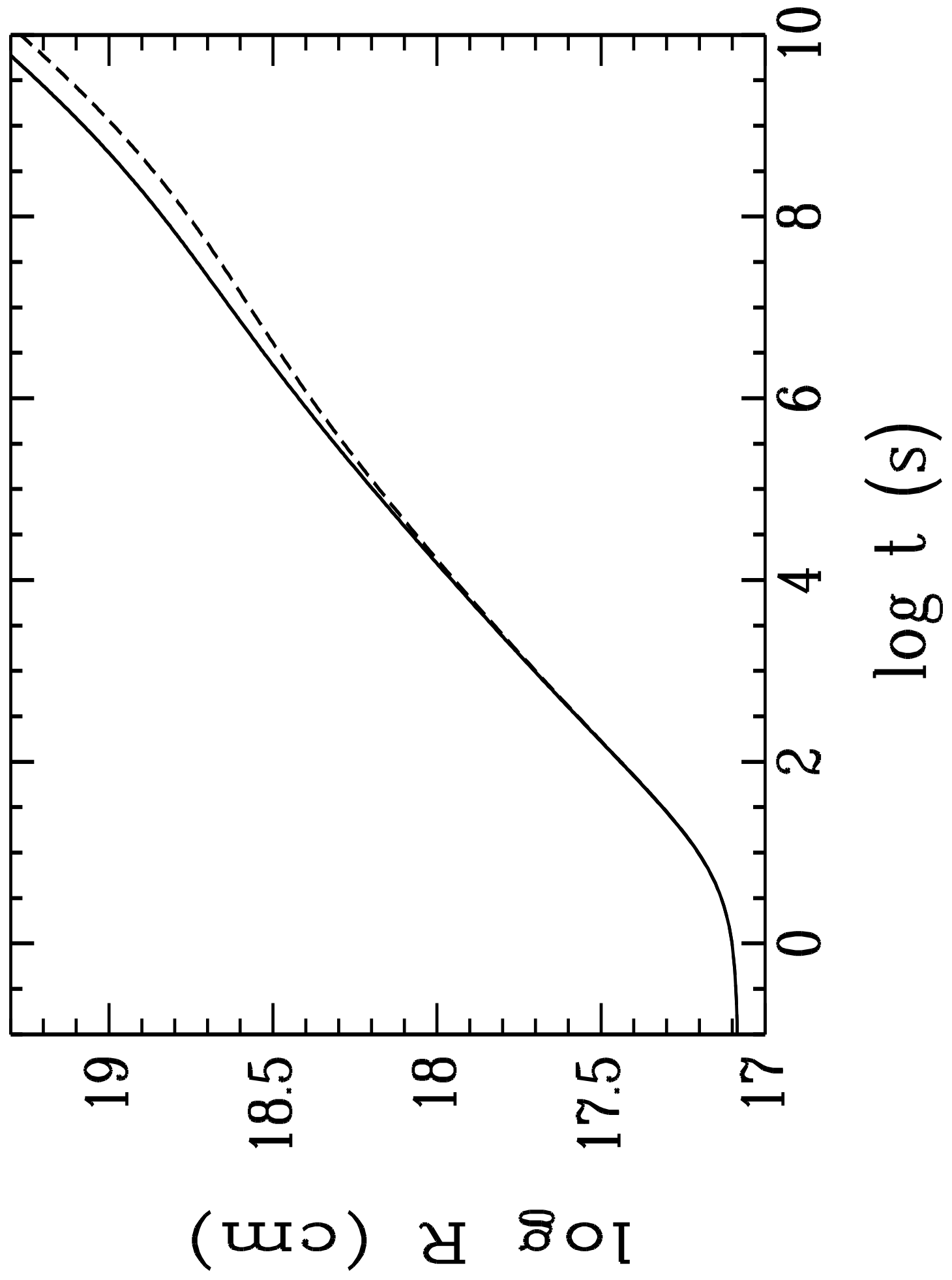




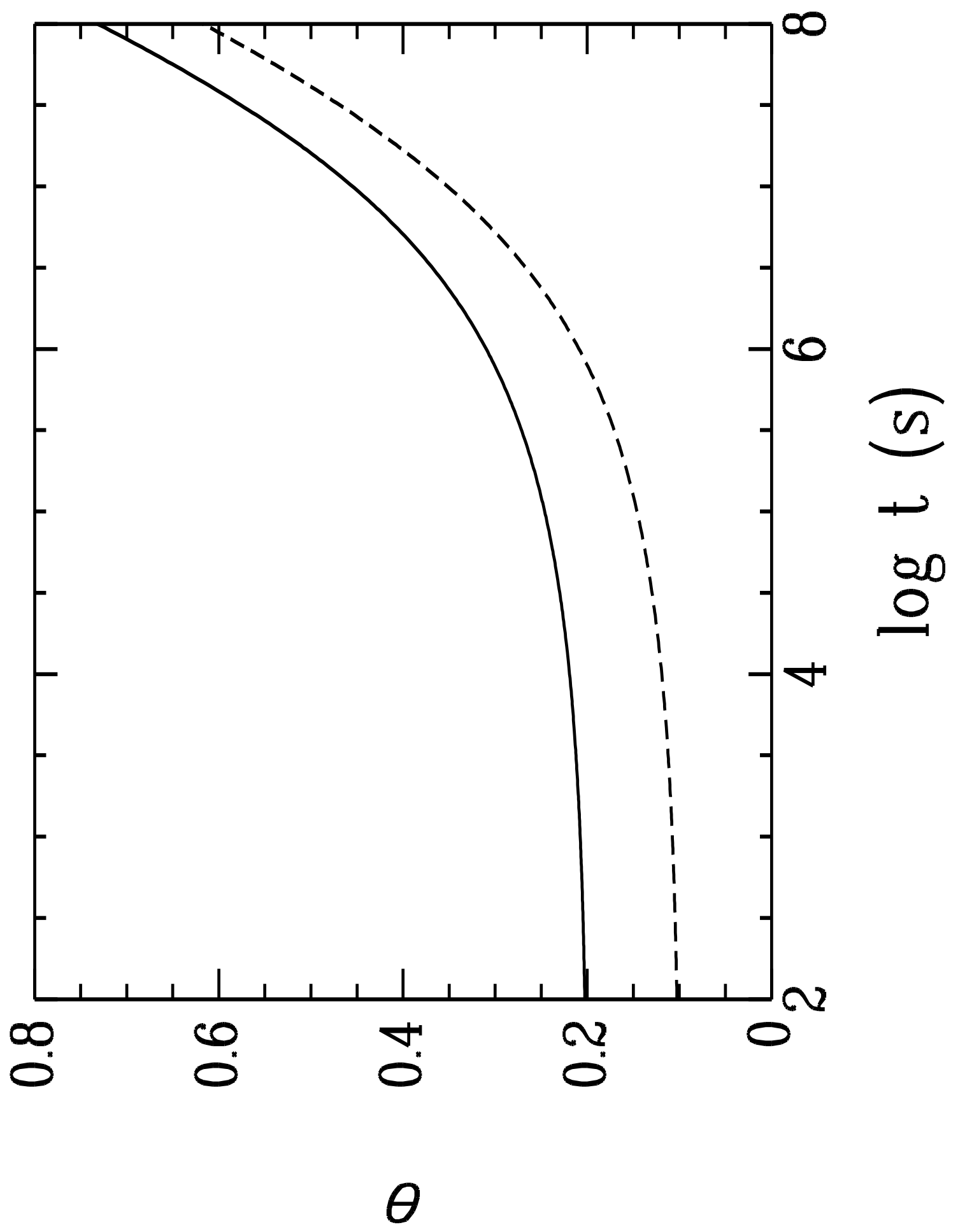




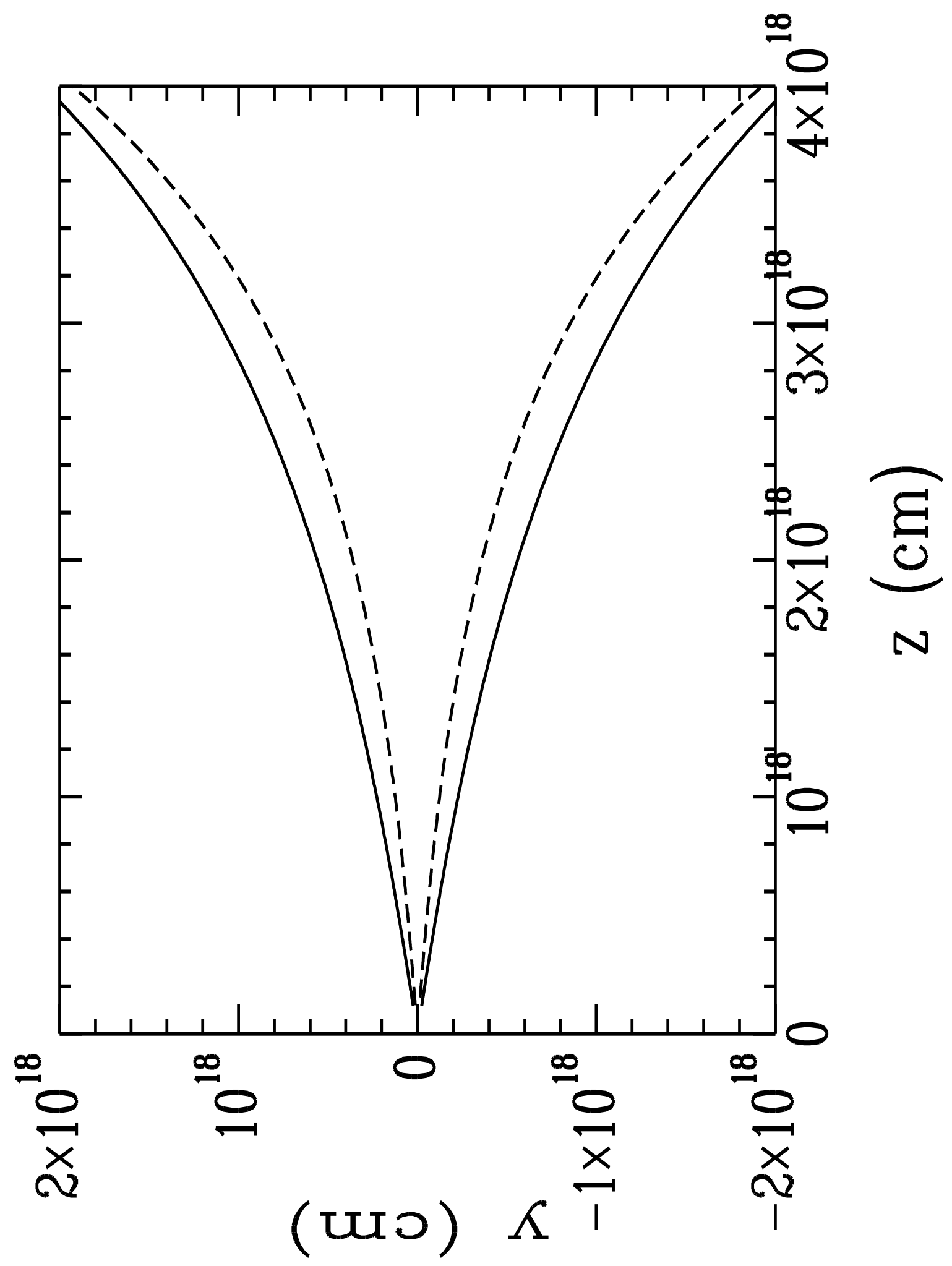




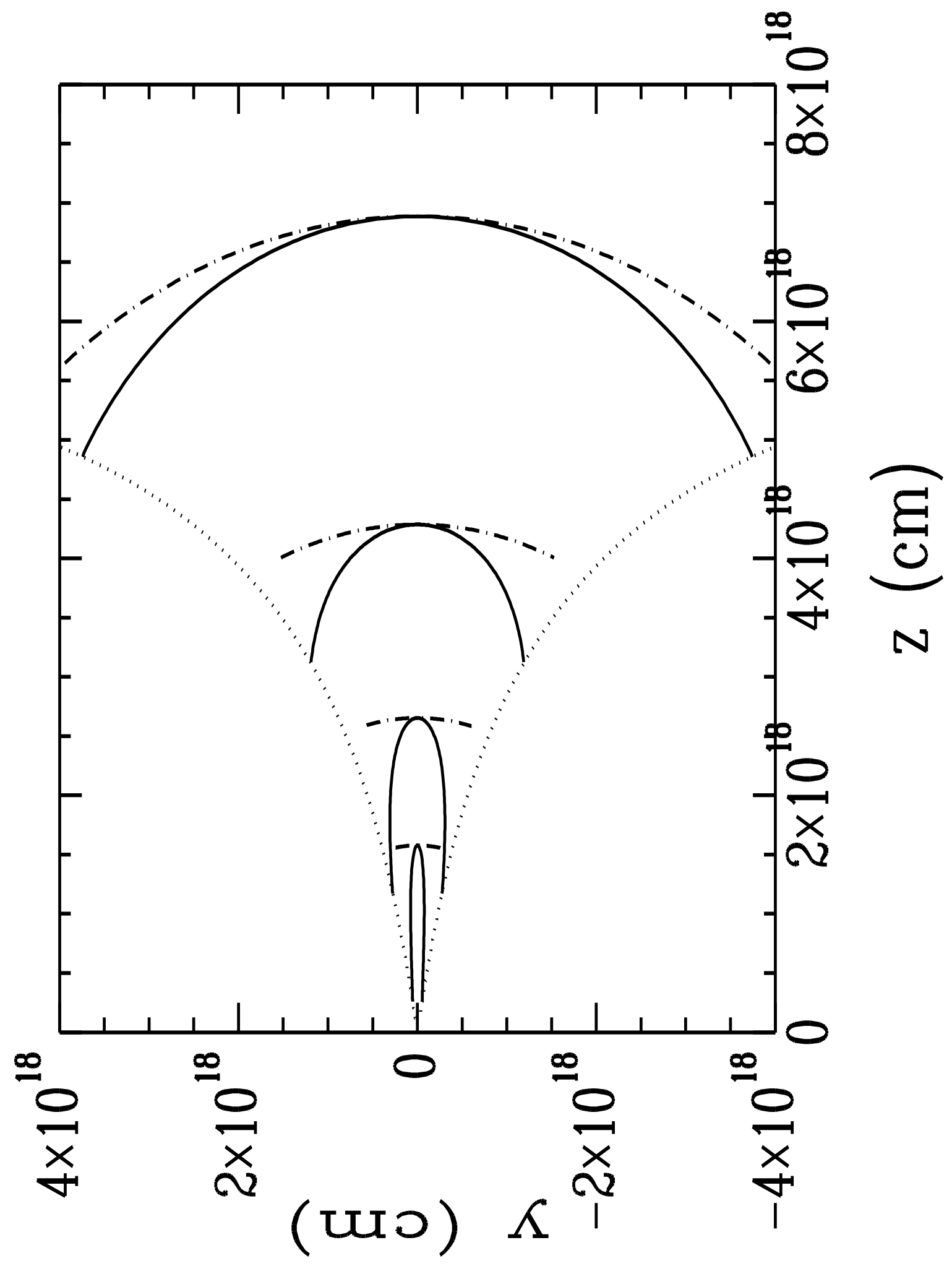




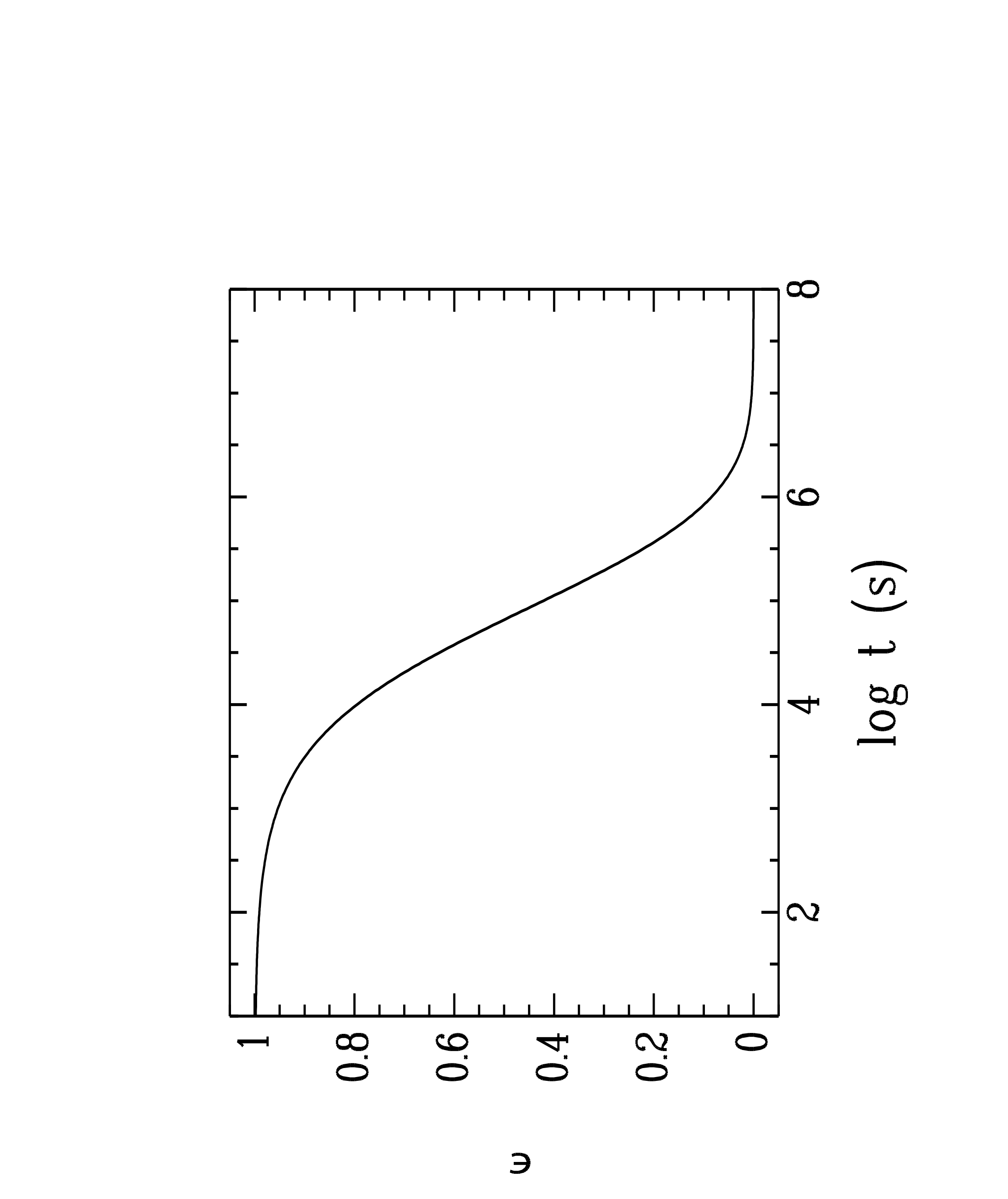




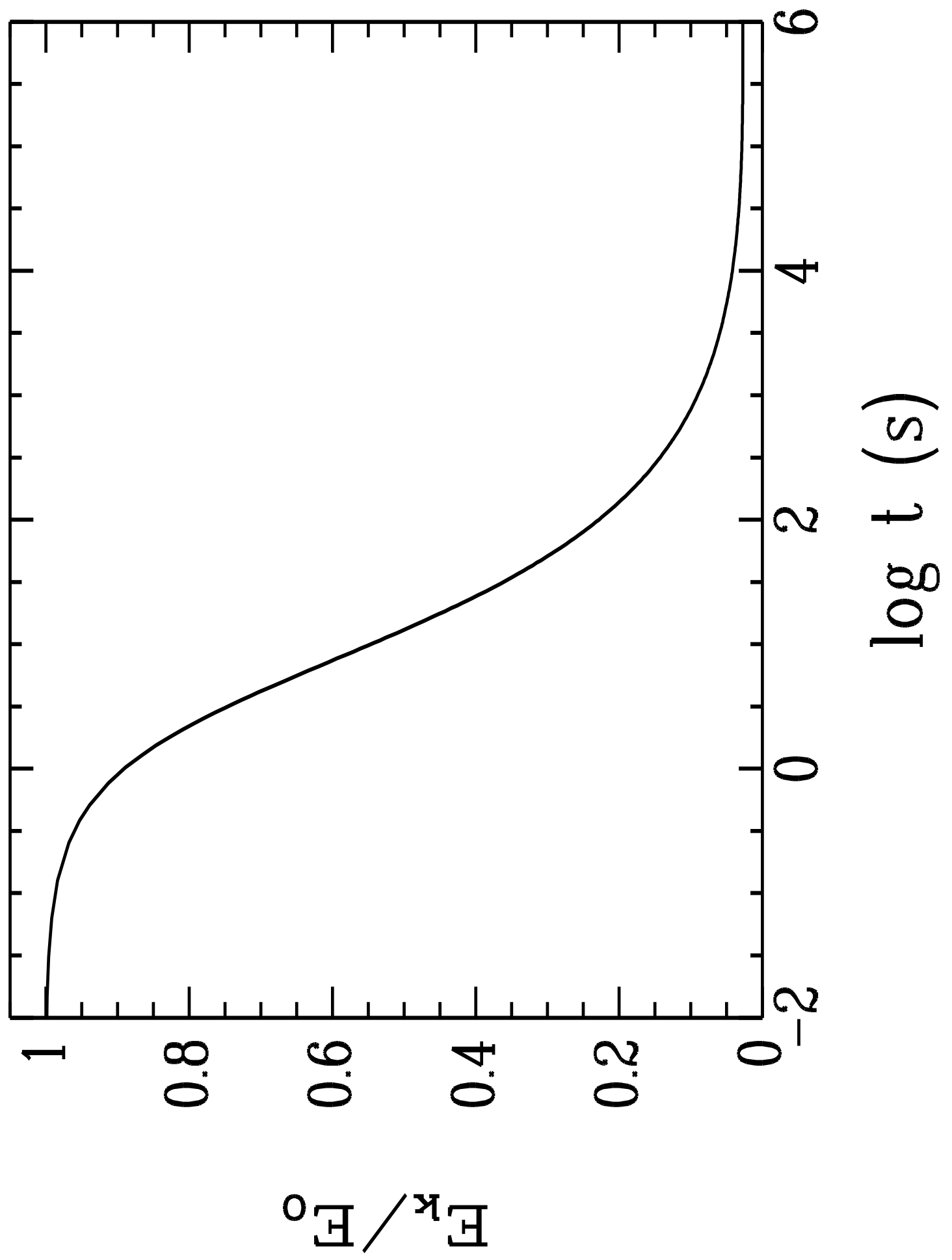




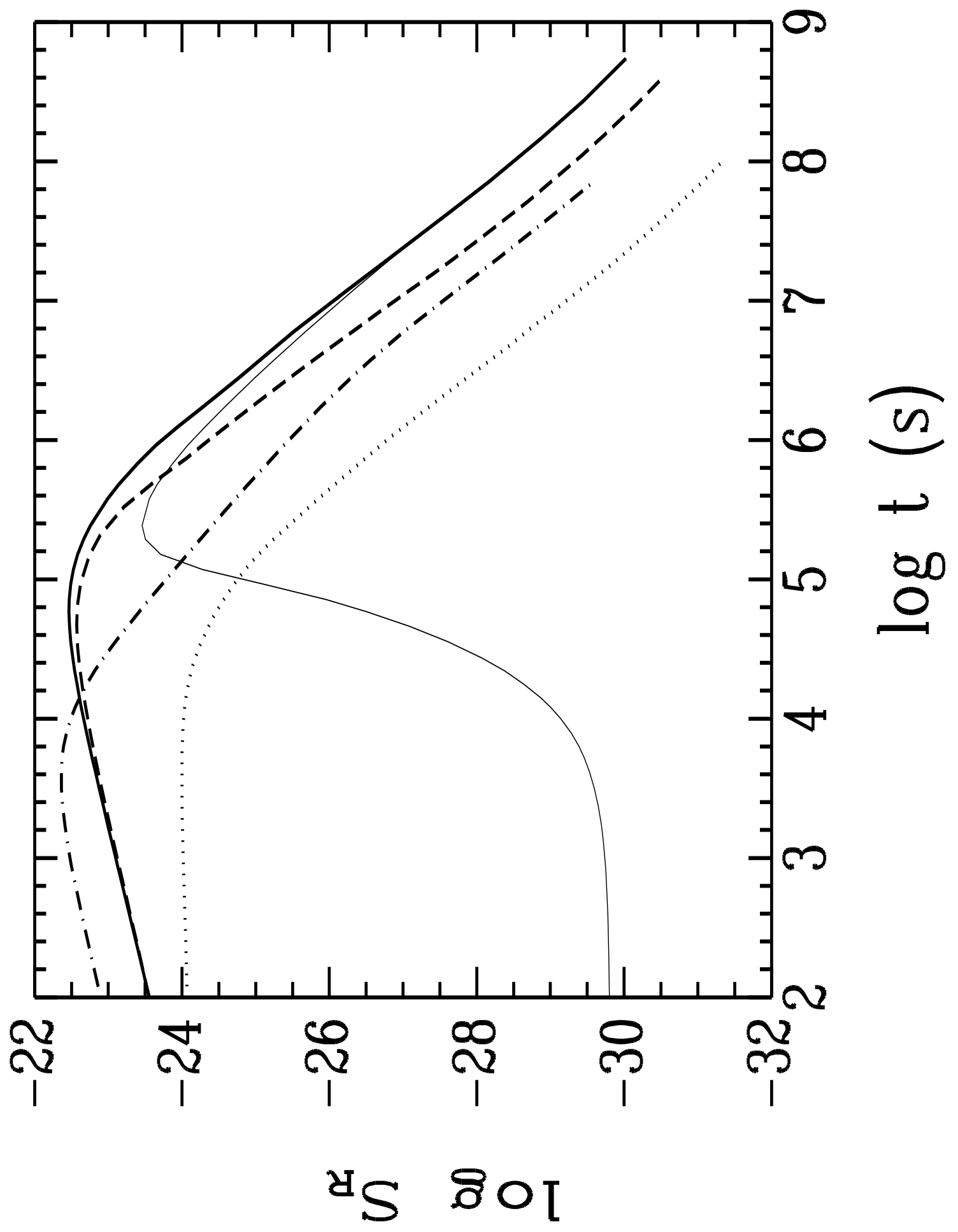

